\title{
Motion Control System Design of Feeding Mechanism of CNC Turret Punch
}

\author{
Cao Aixia ${ }^{1, a^{*}}$, Zhong Peisi $^{2, b}$, Han Yingying ${ }^{3, c}$, Wei Junying $^{4, d}$ \\ 1Qingdao Huanghai University, College of Traffic and Ship Engineering, Shandong Qingdao \\ 266427, China; \\ 2Advanced Manufacturing Technology Center, Shandong University of Science and \\ Technology, Shandong Qingdao, 266590, China; \\ 3 Advanced Manufacturing Technology Center, Shandong University of Science and \\ Technology, Shandong Qingdao, 266590, China; \\ 4 Advanced Manufacturing Technology Center, Shandong University of Science and \\ Technology, Shandong Qingdao, 266590, China) \\ acaoaixia1123@163.com; ${ }^{\text {bpszhong@163.com; } 406548506 @ q q . c o m ; ~}{ }^{\mathrm{d} 916539986 @ q q . c o m}$
}

\begin{abstract}
This paper constructs the hardware of type open CNC system based on the mode of "PC + Motion Control Card "by using DMC1410 PCI motion control card, and studies the use of hardware in the servo control system. Using VC ++6.0 provides the MFC as a tool for secondary exploitation, bottom control procedures were developed. Servo control system software was designed through getting motion function library of motion control card.
\end{abstract}

Keyword: CNC turret punch, Motion control, Servo control system, MFC, Movement function library

\section{Foreword}

Motion control technology is the interdisciplinary comprehensive technology, the core of the electromechanical integration. Position control the implementation of the general MCU or microcomputer directly, but the peripheral circuit structure is complex, need a long development cycle. To control the accuracy and speed of the demand is higher and higher, the traditional control system has not meet the requirements of rapid product development. With the development of control technology, appeared on the market a wide range of motion control card, can satisfy the requirement of the operation speed and high precision control, very suitable for motion control system of CNC equipment development.

In this paper, the motion control system is based on PCI bus PCI - DMC1410 movement control CARDS and function library, PC, control of multiaxial servo motor, and according to the desired trajectory and motion parameters for positioning movement[4]. Has the characteristics of openness, universality, convenient transplantation into all kinds of motion control system development, such as engraving machine, CNC machine tools, and use of MFC for VS2008 provides tools, development and design of CNC turret punch press sheet open buttress feeder of the motion control system. 


\section{Based on the movement control CARDS of feeding mechanism motion control system hardware}

DMC1410B movement control CARDS

DMC1410 impulse type is based on PCI bus motion control card, which can realize up to four step/servo motor control. PCI bus is an advanced high-performance local bus 32/64 bit address data reuse, with plug and play hardware linear interpolation, s-shaped speed control curve, and other functions. Location instructions available single channel pulse (pulse + direction) or dual pulse output (the CW pulse + CCW pulse) way, can a differential or single head type signal. High integration, high reliability, etc.

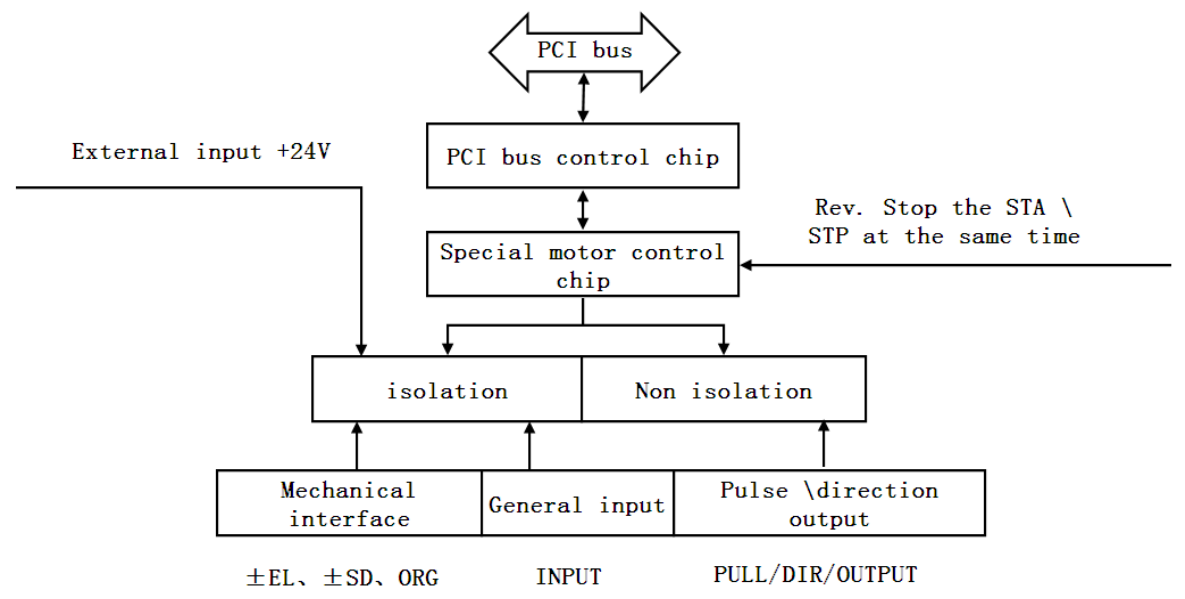

Figure. 1 DMC1410 structure control chart

The movement control CARDS can complete continuous trajectories and little exercise. Speed control and position control is through the point movement. Position control mode includes two deceleration mode: ladder type curve and S type curve acceleration and deceleration deceleration.

The system hardware structure and principle

Sheet pile is the first step of automatic stamping. From preparation workshop to the sheet metal stamping plant, first by the institution of the sheet metal separation device automatically wool embryo buttress material to realize the separation, and then through the sheet metal gripping device - up end to grab. After fetching in a designated place again. Complete the fetching of sheet metal, agency system of buttress and placement, in the process of work requires accurate position and Angle positioning. Need in the process of sheet metal scraping and placed high precision and speed, conventional control system can't satisfy the higher request.

Motion control system hardware is composed of the following four parts: motion control card, PC, servo drive and servo motor. Movement control CARDS for the thunder game technology DMC1410 four axis motion control card. Step families ED - 430 servo drives and + 60 s - 0040-30-4 XXX lg small inertia three-phase servo motor. PC and motion control card together constitute the control unit, the PC main management information flow and data flow, the control system of real-time monitoring and management of the human-computer interaction interface, etc. Control card inserted in the PC motherboard PCI slot. Motion control card dedicated CPU and the PC institutions into a master-slave control structure. 
Automatic lifting of processing, the output of the pulse and direction signals, the origin and limit signals detection can be performed by movement control CARDS. Drive and motion control card of the connection plate is linked together, the encoder speed control, and will receive the pulse signal from the motion control card, through the internal circuit control motor run. Such a one and a half closed loop servo control system. Composition is shown in figure 2.
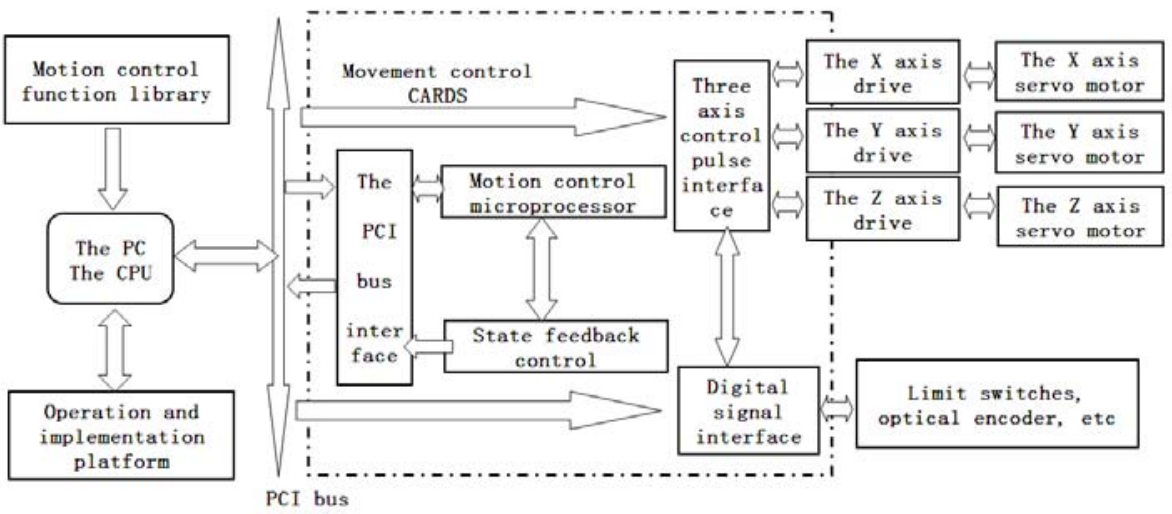

Figure. 2 Movement control system hardware structure diagram

Trapezoidal velocity curve movement patterns

Trapezoidal velocity curve is shown in figure 3. Usually position control in this speed control mode. In motion with constant acceleration in the process of acceleration, and then reaches the specified value to the uniform motion, constant acceleration again after running a certain distance to slow down. Trapezoidal velocity curve, which includes three stages: constant acceleration, uniform and constant deceleration phase.

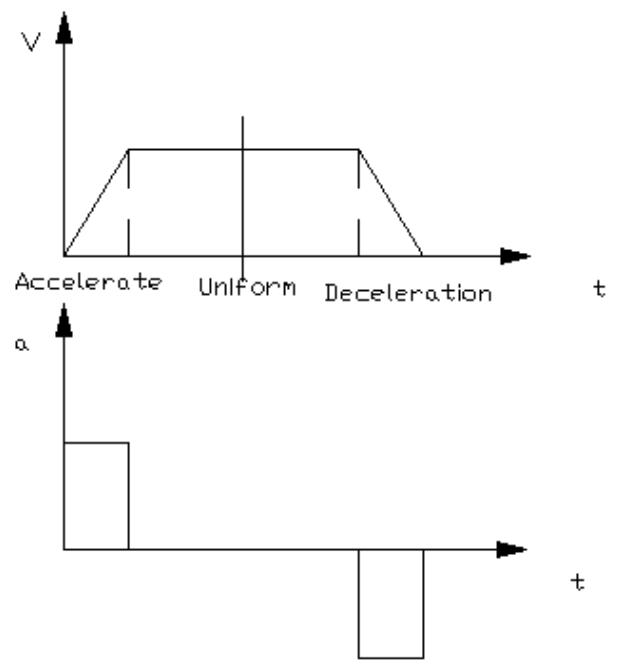

Figure. 3 Trapezoidal velocity and acceleration curve

Speed is with trapezoidal curve changes, mainly because of the effect of inertia. The motor rotor and the dragged object can't be instantly at the specified speed, so there must be certain to accelerate the process. Can be seen from figure 6 trapezoidal curve in the process of acceleration and deceleration, when starting, reach a set speed, came down from the highest speed and the last stop when these four moments, show the speed change and the corresponding acceleration mutation. 
With ladder type curves are the major advantages of the deceleration is in movement process can automatically modify the target position and velocity; The control method and simple calculation, Suitable for the real-time operation; Has a shorter deceleration time. This method is suitable for the control system processing speed is slow, and the occasion of the deceleration process requirements is not high [1].

$\mathrm{S}$ speed curve movement patterns

As shown in figure 4, the acceleration of the s-shaped curve movement model is a continuous process of change. Acceleration or deceleration linear increase or decrease, so in terms of mechanical movement stability is good.

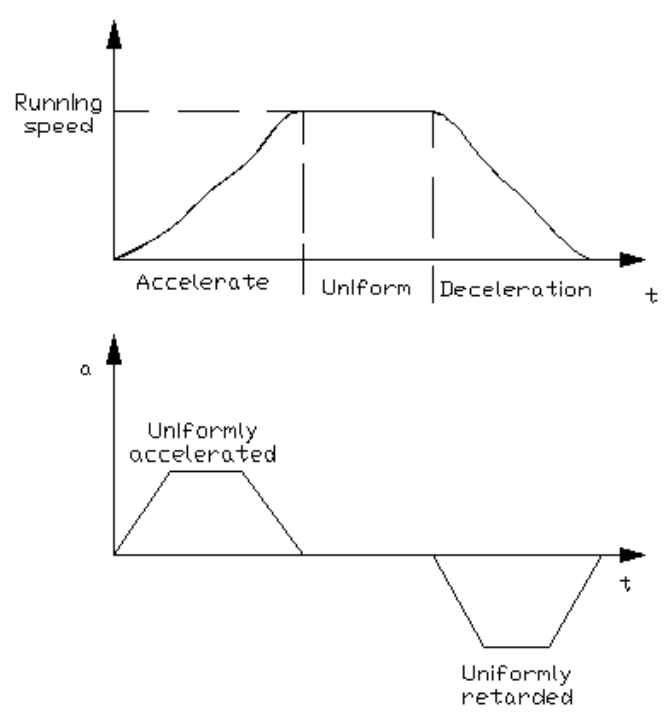

Figure. 4 S velocity and acceleration curve

Trapezoidal deceleration curve can be modified at any time during the process of movement target position and velocity; S curve deceleration can only modify the target location. In the case of acceleration equal to the trapezoidal curve has a shorter deceleration time, and the S curve of movement is smooth, should select the deceleration mode for specific occasions.

\section{Feeding mechanism motion control system software}

The system software design

System software based on Windows platform is based on a preemptive multitasking operating system [], with the development of the friendly graphical interface and abundant resources, to implement multitasking don't need the user intervention. Using modular design, to facilitate system maintenance and upgrade. According to the demand will be divided into the system position module, parameter setting module, motor control module, system management module, information display and anomaly detection process and so on six big modules. VS2008 is a powerful visual programming tool, is currently one of the common application development platform. DMC1410 provide Windows driver, can cut in VS2008 environment with dynamic link libraries, and the application of register level function for developers to provide more convenient, make the development of the underlying easier [3]. Figure 5 for the design of the system software flow chart. 


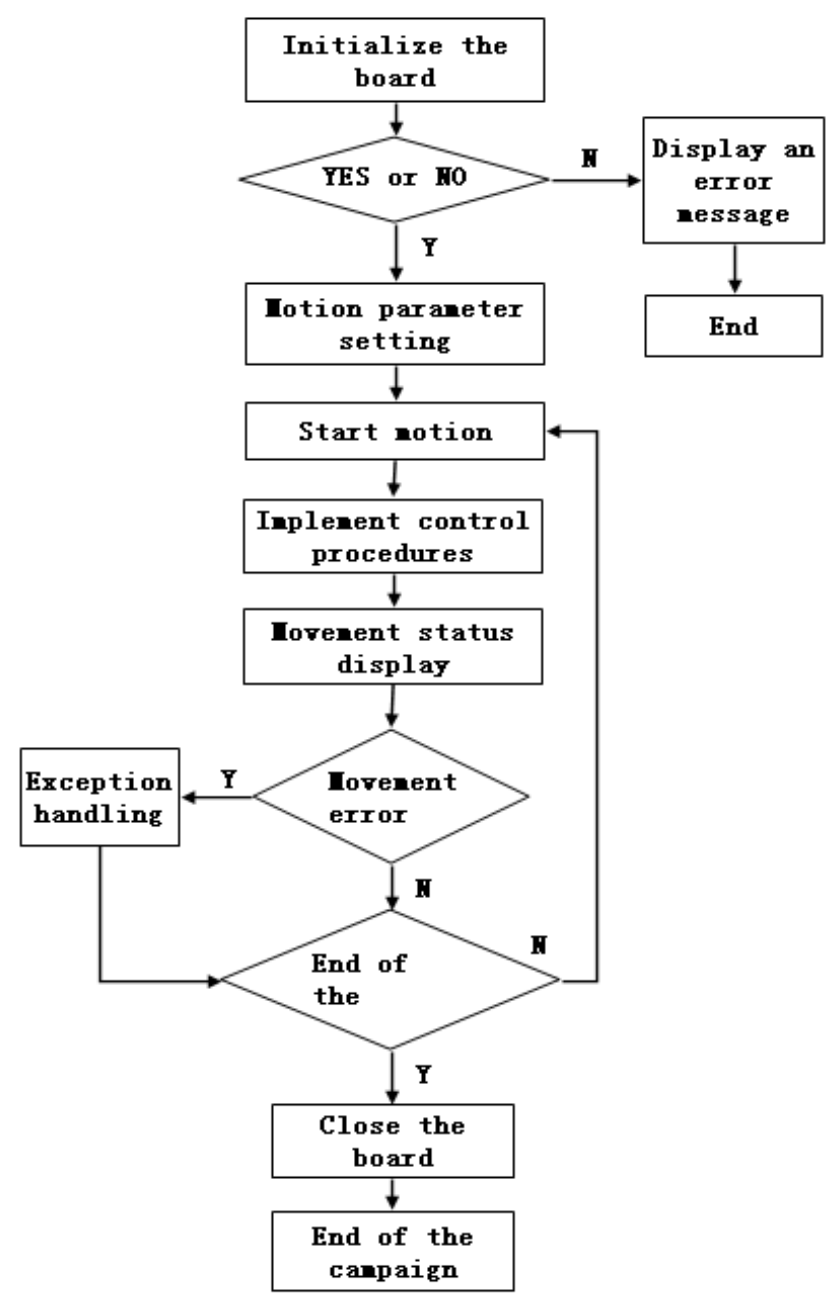

Figure. 5 System software flow chart

Start after the system is initialized, and then set the corresponding operation parameters, parameter setting is mainly responsible for setting feeder $\mathrm{X}, \mathrm{Y}, \mathrm{Z}$ axis motion control pulse output, control the speed of the pulse. After the completion of the parameter Settings, perform motion control module program for motion control, status display module will each axis running state, such as speed, location and other information display. Each function module by mutual coordination control mainframe applications, constitute a complete coordinated motion control system.

Software modular implementation

Software design using modular approach makes feeding machine movement control system software is easy to maintain, and a reconfigurable and scalability. Modular design is in the process of the writing of the program is not detailed step into various statements and control instructions. But with the framework of the main structure and the software process description such as main program, subprogram, sub processes, etc. Will each frame, the input, output, and the links between the definition and debugging, the resulting in a series of algorithm function block as the unit description [4]. Modular design can reduce the complexity of the program, easy to design, debugging and maintenance. Modular development process, must complete the directory structure, naming conventions, global and boundaries of the exception to this rule. Easy to achieve closed independence, repeatability, 
modifiability, uniformity and other higher requirements. The system module and need to pay attention to the following questions: the possibility and necessity of system change; In the aspect of function and structure has the certain independence and integrity; Module cohesion between elements is easy to join and separation. According to modularization design idea on the system module partition the motion control system is divided into the following several modules:

1) the initialization module, to dismantle crib feeding machine movement control system initialization, mainly including interface, motion control card and other parameters initialization.

2) parameter setting module, complete the relevant institutions velocity, acceleration, position of motion are set up. Mold library management, and the design of the servo alarm module.

3) motion control module, mainly including the selection of sports model, receives the limit, switch signals, such as back to zero, the movement control CARDS sent instructions, realize the motion control of the entire crib down the feeding machine.

4) status display, real-time display the current state of the sports information.

5) system management module and anomaly detection processing.

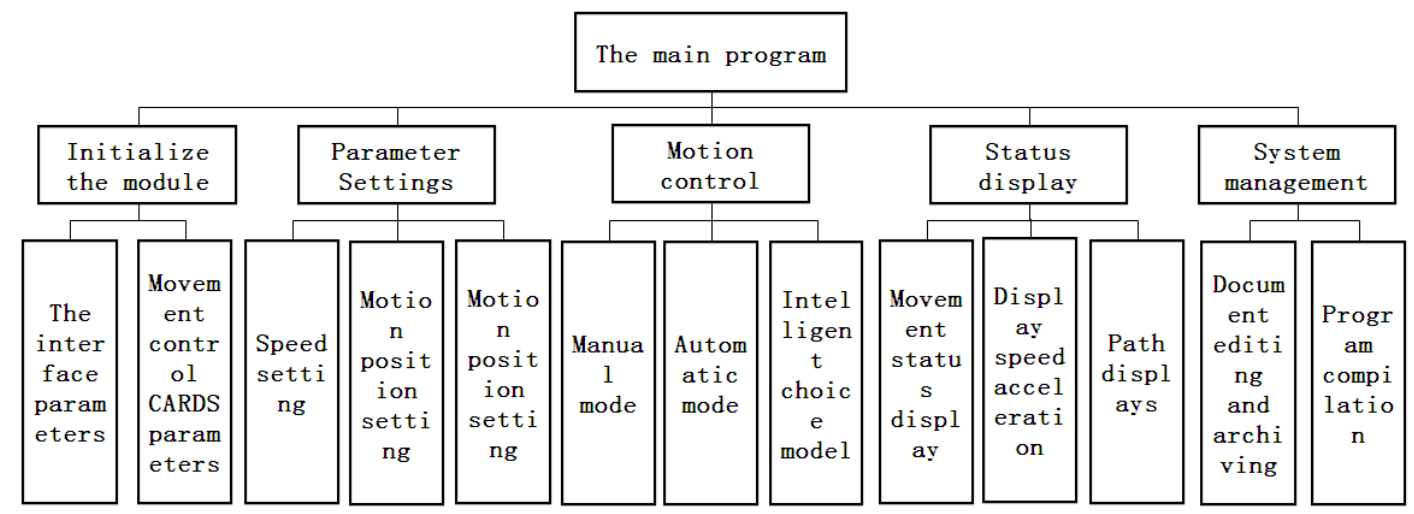

Figure. 6 Motion control system software modules

The implementation of the program

Users can develop the interface corresponding to the requirements of the control system, and call the movement control CARDS on the corresponding part of the function, can be developed to meet the requirements of the motion control system. According to the design of the interface, and to realize the motion control functions, can be done by response function. Dialog box in the $\mathrm{X}, \mathrm{Y}, \mathrm{Z}$ axis three buttons, to the initial speed, acceleration, distance and movement modes related Settings and selective reading.

Implementation process is as follows:

If a continuous movement mode of three axis independence movement, movement function called response function of v_move (axis, str_vel max_vel, Tacc), the end of the campaign calls when v_stop (axis, Tdec), stop the movement of each axis. 


\section{Conclusion}

This article is based on PCI bus PCI - DMC1410 four axis motion control card as the core of the motion control system. In order to overcome the traditional digital motor control device of peripheral circuit is complex, the defects of slow calculation, high speed and high precision motion control is designed. In addition, the system has generality and can be easily transplanted to the development of a variety of motion control system.

\section{Acknowledgements}

This research was financially supported by the natural science fund of Shandong province (ZR2011EEM014) and Science and technology project of Shandong Province (J15LB55) and the project of domestic visiting scholars of young backbone teachers of Shandong Province.

\section{Reference}

[1]. Jianhua, wen-he liao, ru-rong zhou. The study of the several kinds of speed control curve of the CNC system and comparison [J]. Journal of nanjing university of aeronautics and astronautics. 1999, 31 (6) : 706-711.

[2]. Jin Xueyun etc. Visual c + + tutorial [M]. Beijing. Tsinghua university press, 2005

[3]. Zhang jie, Huang Yiqun. Open multi-axis motion control system based on PC development [J]. Journal of servo control. 2007, 47-48

[4]. Yan Jianguo, unmanned aircraft flight control system based on DSP software modular design [J]. Computer measurement and control. 2009, 29 (1) : 2009-221. 